\title{
Sound pressure level calculation methodology investigation of corona noise in AC substations
}

\author{
Xiaowen $\mathrm{Wu}^{1, \mathrm{a}}$, Nianguang $\mathrm{Zhou}^{1}$, Chunming $\mathrm{Pei}^{2}$, Sheng $\mathrm{Hu}^{1}$ and Tao Huang ${ }^{1}$ \\ ${ }^{1}$ State Grid Hunan Electric Power Corporation, Electric Power Research Institute, 410007 Changsha, China \\ ${ }^{2}$ State Grid Electric Power Research Institute, 430074 Wuhan, China
}

\begin{abstract}
In order to improve the measurement accuracy of corona noise in AC substations, the characteristics of different noise sources are measured and analyzed. Wavelet analysis method is employed to decompose the original noisy corona signal. A stop-band comb filter is designed to process the reconstructed approximate coefficient of the decomposed corona noise. The influence of transformer and reactor noises on that of the corona in the substation is eliminated. The speech enhancement technique based on spectral subtraction method is used to extract the pure corona noise form the filtered signal in which the cooling-device noise in included. The proposed method is applied to process field corona noise in a UHV substation. The 1/3-Octave spectrum and the A-weighted sound pressure level of the purified corona noise are calculated.
\end{abstract}

Keywords: corona noise; AC substations; comb filter; spectral subtraction.

\section{Introduction}

In AC substations, the main power transformer, high voltage (HV) reactor and corona generated on the power transmission structure are the three main audible noise sources [1-2]. It has been proved that sound pressure level (SPL) of the power apparatus in AC substations increases as the voltage level increases. Corona noise is gradually becoming the concerning focus of people in these years for the increasing construction scale of power grid. Accurate SPL measurement of the corona noise provides reference for the noise assessment and control. However, direct on-site SPL measurement of the corona noise is a challenging task for the complex background audible noises in substations.

In most cases, corona noise measurement is carried out with experiment [3-7]. Traditional field test method of corona noise is mainly based on FFT, which is the mixed result of corona and background noises [5]. Usually, some common frequency components exist in the corona and background noises. In this case, the test result of corona characteristics will be largely affected if a single FFT-based method was employed.

Noise characteristics of the main power apparatus in AC substations are analyzed in this paper. Based on the characteristic discrepancy, time- and frequency-domain methods are combined to obtain the pure corona noise. The wavelet analysis and spectral subtraction methods are used. In order to calculate the 1/3-Octave spectral, 1/3-Octave filters are designed. The A-weighted SPL of the obtained pure corona noise is calculated by logical add operation of the $1 / 3$-Octave spectral.

\footnotetext{
${ }^{a}$ Corresponding author : wxwwhu@163.com
} 


\section{Noise characteristics analysis}

According to the available investigations, audible noises generated by power transformers and HV reactors have identical spectral below $2 \mathrm{kHz}$, in which the $50 \mathrm{~Hz}$ and its harmonics are the dominant components [8]. Transformer noise has two distinct components. The most dominant component is the humming noise which is originated from magnetic forces on windings and magnetostriction of the core. The secondary one is the noise associated with the cooling fans, which is characteristically broad-band in nature [6]. Figure 1 gives the noise spectrum tested on-site of a power transformer and a HV reactor. Figure 2 gives the spectrum of corona noise.

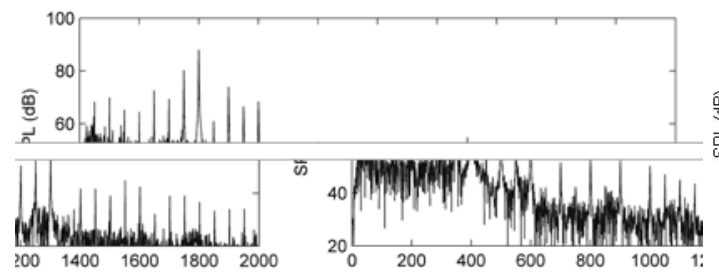

(a)

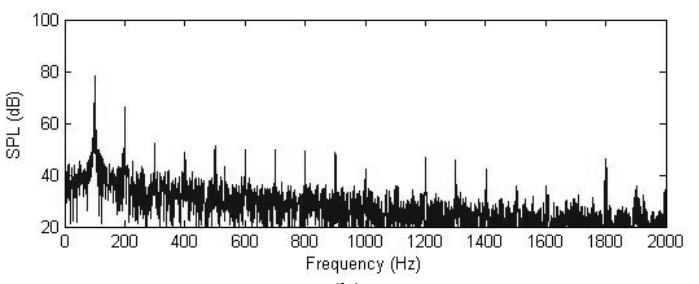

(b)

Figure 1. Noise spectrum distribution of a power transformer (a) and a HV reactor (b)

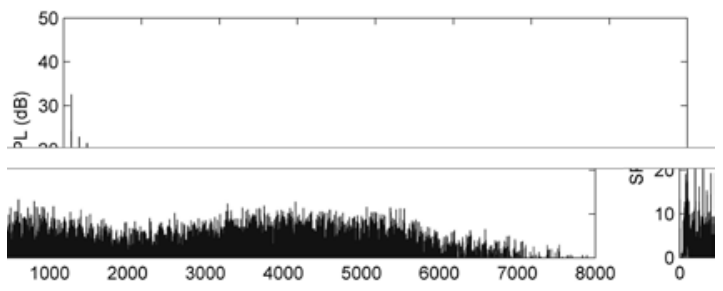

Figure 2. Spectrum distribution of corona noise

It can be seen in Figure 1 that the dominant frequency components of the transformer and reactor noises are $400 \mathrm{~Hz}$ and $100 \mathrm{~Hz}$, respectively. Although the spectra of the power transformer and reactor are apparently different in distributions, a common property can be observed that the power transformer and reactor noises are mainly in the frequency-bands of $50 \mathrm{~Hz}$ and its harmonics. It makes the methodology practicable that within the frequency of $2 \mathrm{kHz}$ the power transformer and reactor noises can be filtered from the measured corona noise by designing proper stop-band comb filters.

As shown in Figure 2, the corona noise spectrum is distributed in a wide range up to $8 \mathrm{kHz}$. As low frequency noises from the power transformers and reactors can propagate far distances, thus some prominent frequency components at $50 \mathrm{~Hz}$ and its harmonics within $500 \mathrm{~Hz}$ can be observed in the spectrum, which means that the measured corona noise is influenced by that of the transformer and reactor. In order to obtain pure corona noise, the corona noise measured on-site needs to be preprocessed.

\section{SPL calculation algorithm}

The SPL calculation process of the pure corona noise is shown in Figure 3. The influence of transformer body noise can be eliminated with a designed stop-band comb filter. The noise frequency of the cooling device is usually below $2 \mathrm{kHz}$, in which range the corona noise has similar spectral distribution. Therefore, the pure corona noise cannot be obtained with direct frequency-domain analysis. The cooling device noise waveform amplitude is stable in time domain, no peaks and period cycles can be obviously observed. In comparison, the waveform of corona noise is pulsating. In this case, the speech enhancement technique based on spectral subtraction is utilized, with which the pure corona noise can be extracted. 1/3-Octave filters are designed and used to divide the spectrum of the 
pure corona noise into several sections. In each section, the SPL is calculated. All the obtained SPLs are finally used to calculate the equivalent A-weighted SPL of the pure corona noise.

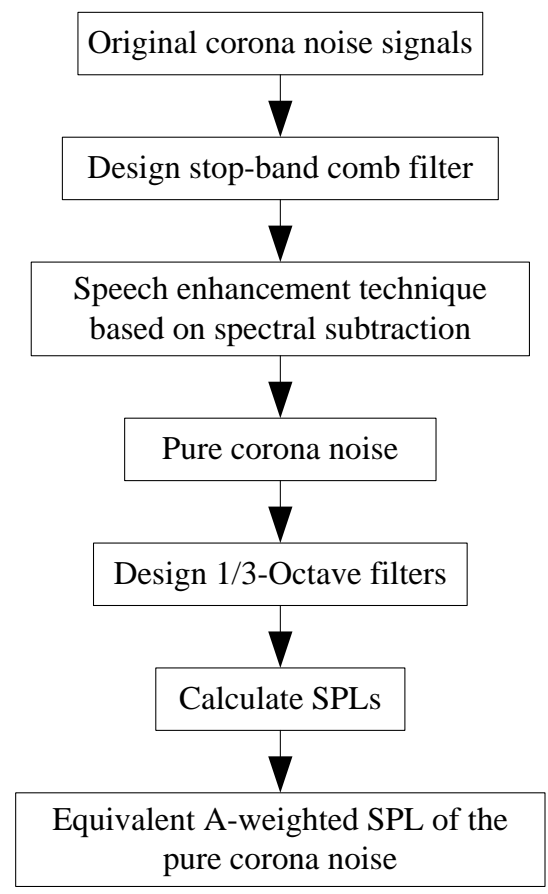

Figure 3. Flow chart of the SPL calculation methodology

\subsection{Wavelet analysis}

Wavelet are defined as a family of functions derived from a function called a mother wavelet $(\psi)$ indexed by two parameters, scale factor $(a)$ and translation or time shift factor $(b)$ in time [9-10].

$$
\psi_{a, b}(t)=\frac{1}{\sqrt{|a|}} \psi\left(\frac{t-b}{a}\right) \quad a, b \in \mathbf{R} ; a \neq 0
$$

where $\psi_{a, b}(t)$ denotes the daughter wavelets derived from mother wavelet $\psi(t)$ by contineous varing $a$ and $b$.

For the above family of functions, the continuous wavelet transform is defined as follows:

$$
C W T(a, b)=<x(t) \cdot \psi_{a, b}(T)>=|a|^{-1 / 2} \int_{R} x(t) \psi^{*}\left(\frac{t-b}{a}\right) \mathrm{d} t
$$

where $<\cdot>$ indicates the inner product, '*' stands for complex conjugate.

In order to improve the calculation efficiency of wavelet analysis, wavelet transform has a digitally implemented format called Discrete Wavelet Transform (DWT), which is defined as

$$
D W T[i, j]=a_{0}^{-\frac{i}{2}} \sum_{n} x[n] \psi\left[\frac{j-n a_{0}^{i}}{a_{0}^{i}}\right]
$$

where $\psi(n)$ is the mother wavelet, $i$ and $j$ are integers, $a_{0}^{i}=a, n a_{0}^{i}=b$. 


\subsection{Stop-band comb filter design}

According to the characteristics of the body noise, a comb filter at the stop band of $50 \mathrm{~Hz}$ and its harmonics is designed with a direct-form II transposed structure, which can be expressed as follows:

$$
y(k)=b_{0} x(k)+b_{1} x(k-1)+\cdots+b_{m} x(k-m)-a_{1} y(k-1)-\cdots-a_{n} y(k-n)
$$

where $k$ - 1 is the filter order, $n$ is the feed-back filter order, $m$ is the feed-forward filter order, $a_{i}$ and $b_{i}$ are the feed-back and feed-forward filter coefficients, respectively.

A 6-order stop-band comb filter is used in the separation process, as shown in Figure 4. The bandwidth of both filters is chosen to be $10 \mathrm{~Hz}$ referenced to the $-3 \mathrm{~dB}$ level.

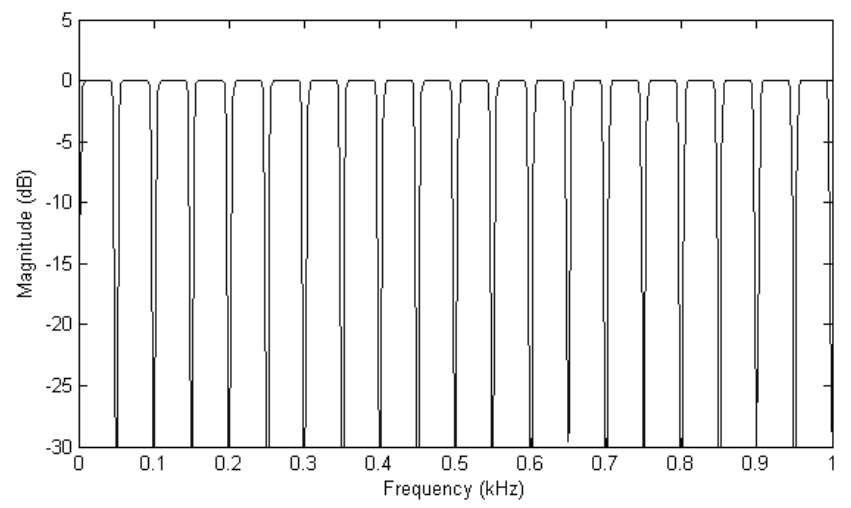

Figure 4. Amplitude-frequency response of the stop-band comb filter

\subsection{Speech enhancement technique based on spectral subtraction}

Spectral subtraction is an effective algorithm to process wide-band noise such as cooling-device and corona noises [11]. The principle diagram of spectral subtraction is shown in figure 5.

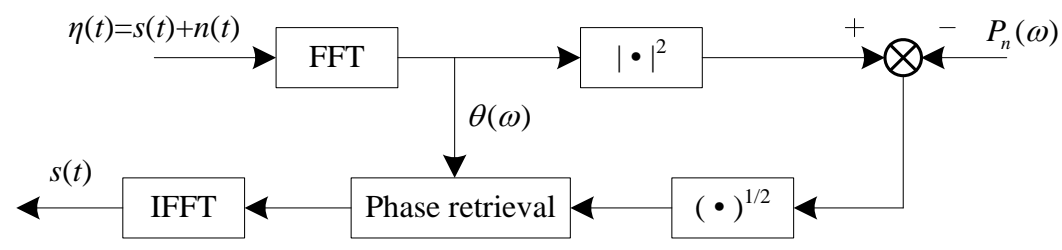

Figure 5. Principle diagram of spectral subtraction.

Assuming that $n(t)$ is the cooling-device noise and $s(t)$ is the corona noise, the noisy signal $\eta(t)$ is the sum of $n(t)$ and $s(t), \eta(t)=n(t)+s(t)$. $t$ denotes the sampling time. $s(t)$ and $n(t)$ are further assumed to be independent statistically. The FFT of $\eta(t)$ can be written as

$$
I(\omega)=S(\omega)+N(\omega)
$$

where $S(\omega)$ and $N(\omega)$ are the FFT of $s(t)$ and $n(t)$, respectively.

As $s(t)$ and $n(t)$ are independent, thus the power of noise signals can be computed as [12]

$$
P_{\eta}(\omega)=P_{s}(\omega)+P_{n}(\omega)=|S(\omega)|^{2}+|N(\omega)|^{2}
$$


where $P_{\eta}(\omega), P_{s}(\omega)$ and $P_{n}(\omega)$ are the power spectra of signals $\eta(t), s(t)$ and $n(t)$, respectively.

The cooling-device noise keeps stationary before and when the corona noise occurs. Therefore, the silent part of the cooling-device noise can be used for power spectral estimation of the corona noise.

$$
P_{s}(\omega)= \begin{cases}P_{\eta}(\omega)-P_{n}(\omega), & P_{\eta}(\omega) \geq P_{n}(\omega) \\ 0, & P_{\eta}(\omega)<P_{n}(\omega)\end{cases}
$$

Only the power spectral transform is considered in (7). As human ear is not sensitive to the phase of noise signal, the phase spectrum of corona noise is substitute with that of the noisy signal. The corona noise in time domain can be expressed as

$$
s(t)=\operatorname{IFFT}\left(\sqrt{P_{s}(\omega)} \cdot \exp (\mathrm{j} \theta(\omega))\right.
$$

where $\theta(\omega)$ is the phase spectrum of the noisy signal.

Subtracting corona noise from the noisy signal, the cooling-device noise is obtained.

$$
n(t)=\eta(t)-s(t)
$$

\subsection{SPL calculation method}

The effective sound pressure $p_{\mathrm{e}}$ is defined as the root mean square of the sound pressure in the time interval $T$, which can be expressed as [5]

$$
p_{\mathrm{e}}=\sqrt{\frac{1}{T} \int_{0}^{T} p^{2} d t}
$$

SPL is usually employed to assess the noise level, which is defined by the following equation:

$$
L_{\mathrm{p}}=20 \lg \left(p_{\mathrm{e}} / p_{0}\right)
$$

where $p_{0}=2 \times 10^{-5} \mathrm{~Pa}$ is the reference pressure.

In the spectral analysis process of corona noise, obtaining detail spectral distribution in all frequency components is not necessary. The spectrum is usually divided into several connected sections, each section is called octave, in which the sound power is assumed to be uniform. One of the most used bandwidth of each section is $1 / 3$ Octave. In 1/3 Octave, the relationship of centre frequency $f_{0}$, upper cut-off frequency $f_{\mathrm{h}}$ and lower cut-off frequency $f_{\mathrm{l}}$ can be written as:

$$
\begin{gathered}
f_{0}=\sqrt{f_{\mathrm{h}} f_{\mathrm{l}}} \\
f_{\mathrm{h}}=2^{1 / 3} f_{\mathrm{l}}
\end{gathered}
$$

The $1 / 3$ Octave analysis of corona noise is actually a process using different pass-band filters to deal with its corresponding octave. According to the standard GB/T 3241-2010, the 6-order digital Butterworth filter is used to design the 1/3-Octave filters. The normalized upper cut-off frequency $w_{2}$ and lower cut-off frequency $w_{1}$ can be determined, respectively, by the following equations:

$$
w_{1}=\frac{2 f_{0}}{\alpha F_{s}}, \quad w_{2}=\frac{2 \alpha f_{0}}{F_{s}}
$$




$$
\begin{gathered}
\alpha=\frac{\left(1+\sqrt{1+4 Q_{\mathrm{d}}^{2}}\right)}{2 Q_{\mathrm{d}}} \\
Q_{\mathrm{d}}=\frac{f_{0}}{f_{\mathrm{h}}-f_{\mathrm{l}}} \frac{\pi}{N \sin \left(\frac{\pi}{N}\right)}
\end{gathered}
$$

where $F_{\mathrm{s}}$ is the sampling frequency, $N$ is the filter order.

The transfer function of digital Butterworth filter can be written as:

$$
H(z)=\frac{b_{1}+b_{2} z^{-1}+\cdots+b_{N+1} z^{-N}}{1+a_{2} z^{-1}+\cdots+a_{N+1} z^{-N}}
$$

Considering the weight of the octave, the A-weight SPL of the filtered noise with centre frequency $f_{0}$ can be calculated with (10) and (11).

\section{Corona noise analyses results and discussion}

The corona noise is measured on-site in a $1000 \mathrm{kV}$ UHV substation. The sampling frequency is 44.1 $\mathrm{kHz}$. As the spectra of the power transformers and reactors are below $2 \mathrm{kHz}$, and in order to avoid that some useful harmonic frequencies of $50 \mathrm{~Hz}$ above $2 \mathrm{kHz}$ is filtered, the measured noisy corona signal is decomposed by wavelet analysis method. Only the reconstructed approximate coefficient is filtered. The coif5 wavelet is chosen to be the wavelet base function. 5-layer wavelet decomposition has been conducted, and the result is given in Figure 6. S1 is the original measured noisy corona signal in Pa. a5 is the reconstructed approximate coefficient of S1. d1, d2, d3, d4 and d5 are the reconstructed detail coefficient of each layer. a5 is in the frequency range of $0 \sim F_{s} / 32$, in which the body noise of power transformers and reactors are included. It can be seen that the corona noise is mostly distributed in the frequency range above $1 \mathrm{kHz}$.

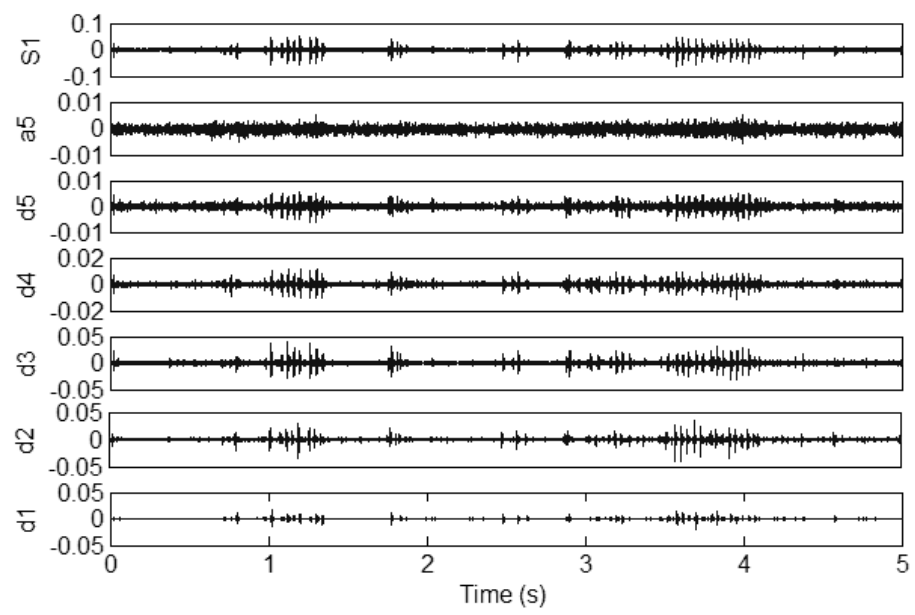

Figure 6. Wavelet decomposition of the noisy corona signal 

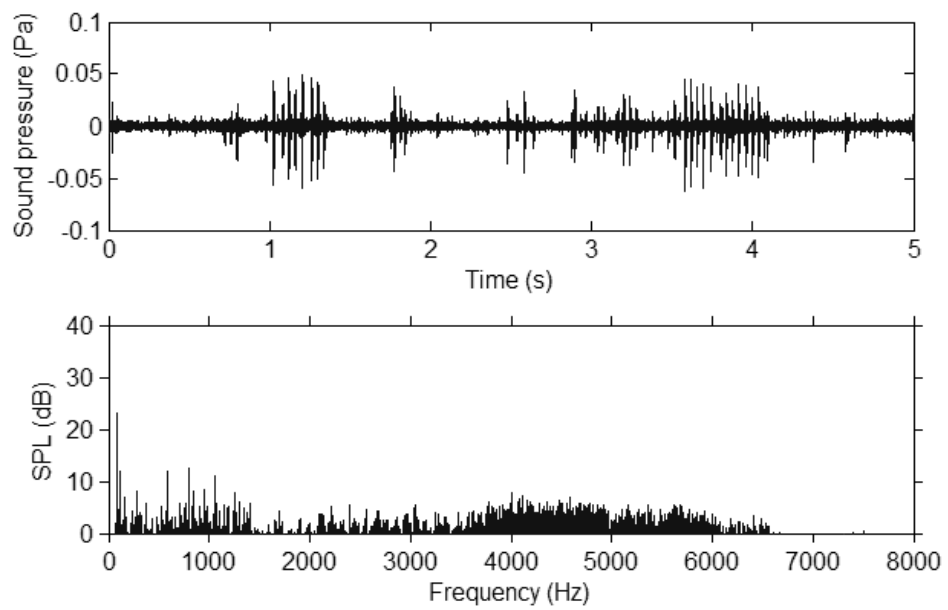

Figure 7. Filtered corona noise signal

A stop-band comb filter of $50 \mathrm{~Hz}$ and its harmonics as shown in Figure 4 is designed to filter the reconstructed noise signal a5. The filtered result is given in Figure 7. It can be found that the body noise of power transformers and reactors at the frequencies of $50 \mathrm{~Hz}$ and its harmonics has been successfully removed from the reconstructed approximate coefficient of the noisy corona signal.

Cooling-device usually generates steady noise which has wide frequency band and is still included in the filtered corona noise. Unlike the cooling-device, the noise generated by corona is changing with time and has pulsating waveform in time domain. Based on this discrepancy, the speech enhancement technique based on spectral subtraction is employed to process the mixed corona and cooling-device noise signals. Assuming the corona noise is the speech signal, the mixed signal is divided into 2047 frames. Each frame has 256 data points. 128 data points are overlaped between two sequential frames. As no corona occurs in the 15 to 18th frames, signals in these frames can be assumed to be the cooling-device noise. Applying the spectral subtraction method, the pure corona noise is obtained, as shown in Figure 8.

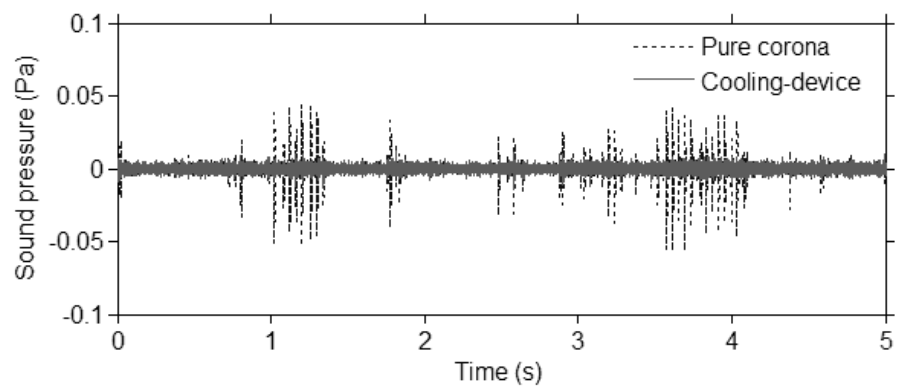

Figure 8. Pure corona noise signal 


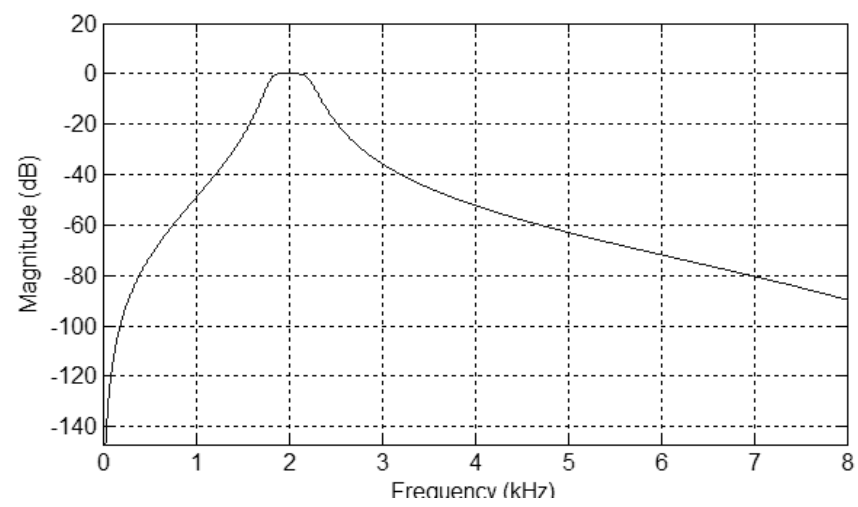

Figure 9. Magnitude response of 1/3-Octave filter at $2 \mathrm{kHz}$

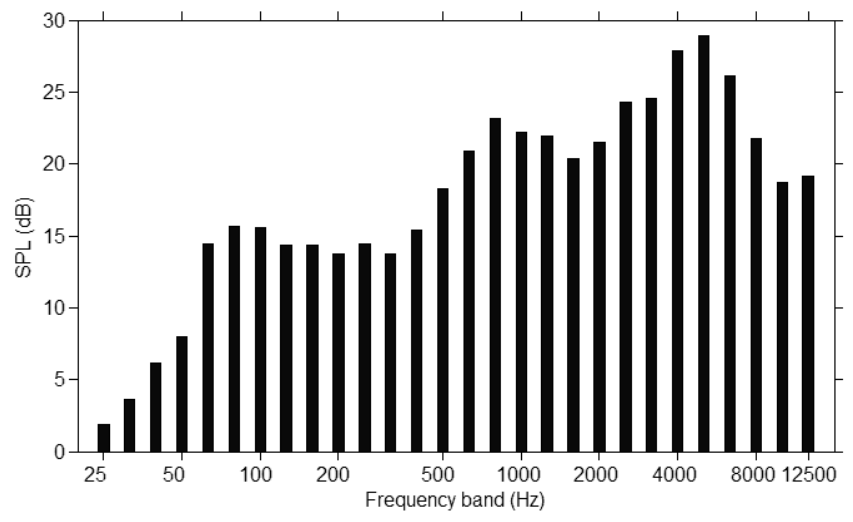

Figure 10. 1/3-Octave distribution of the pure corona noise

Twenty eight 1/3-Octave filters in all are designed to analyze the octave distribution of the pure corona noise. The magnitude response of one filter at $2 \mathrm{kHz}$ is given in Figure 9. The 1/3-Octave distribution of the pure corona noise is shown in Figuire 10. It can be found that high frequencies over $1 \mathrm{kHz}$ are the main components of pure corona noise. Applying the proposed algorithm to the original measured noisy corona signal and pure corona noise, the equivalent A-weight SPL are $40.4 \mathrm{~dB}(\mathrm{~A})$ and $38.7 \mathrm{~dB}(\mathrm{~A})$, respectively. The SPL of pure corona noise is 1.7 decibels lower than that of the noisy signal. It is proved that the effect of transformer and reactor noises in AC substaion is not neglectable on the field corona noise measurement.

\section{References}

1. B. Zhou, C. Pei, Y. Ni, J. Zhang, High Voltage Engineering, 39, 6(2013)

2. B. Wan, X. Wu, Y. Yang, High Voltage Engineering, 32, 3(2006)

3. K. Tanabe, International Journal of Quantum Chemistry, 125, 1(1998)

4. K. Tanabe, IEEJ Transactions on Fundamentals \& Materials, 129, 8(2009)

5. T. Lu, D. Zhang, X. Li, High Voltage Engineering, 41, 3(2015)

6. J. Tang, J. He, Y. Liu, Proceedings of the CSEE, 30, 4(2010)

7. Y. Chen, G. Zhan, C. Zhou, High Voltage Engineering, 35, 8(2009)

8. R.S. Ming, J. Pan, M.P. Norton, S, Applied Acoustics, 56, 1(1999)

9. P. Kang, D. Birtwhistle, IEEE Transactions on Power Delivery, 16, 3(2001)

10. P.W. Tse, W.X. Yang, H.Y. Tam, Journal of Sound and Vibration, 277, 1(2004)

11. I. Cohen, B. Berdugo, IEEE Signal Processing Letters, 9, 1(2002)

12. R. Martin, IEEE Transactions on Speech and Audio Processing, 9, 5(2001) 